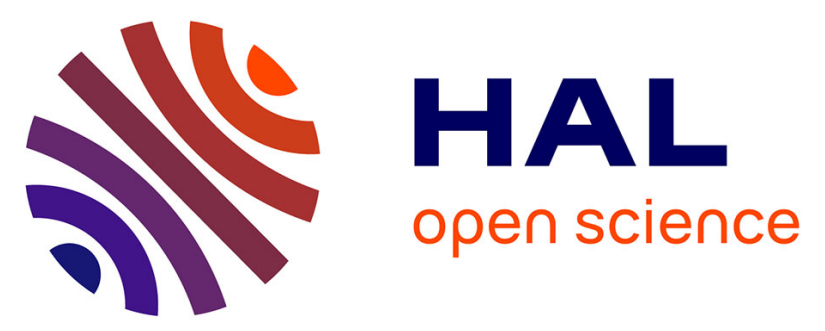

\title{
High Frequency Oscillations and spikes running down after SEEG-guided thermocoagulations in the epileptogenic network of periventricular nodular heterotopia
}

\author{
Julia Scholly, Francesca Pizzo, Alexander Timofeev, Maria Paola \\ Valenti-Hirsch, Irène Ollivier, François Proust, Nicolas Roehri, Edouard \\ Hirsch, Christian G. Bénar, Fabrice Bartolomei
}

\section{To cite this version:}

Julia Scholly, Francesca Pizzo, Alexander Timofeev, Maria Paola Valenti-Hirsch, Irène Ollivier, et al.. High Frequency Oscillations and spikes running down after SEEG-guided thermocoagulations in the epileptogenic network of periventricular nodular heterotopia. Epilepsy Research, 2019, 150, pp.27-31. 10.1016/j.eplepsyres.2018.12.006 . hal-03586405

\section{HAL Id: hal-03586405 https://hal.science/hal-03586405}

Submitted on 23 Feb 2022

HAL is a multi-disciplinary open access archive for the deposit and dissemination of scientific research documents, whether they are published or not. The documents may come from teaching and research institutions in France or abroad, or from public or private research centers.
L'archive ouverte pluridisciplinaire HAL, est destinée au dépôt et à la diffusion de documents scientifiques de niveau recherche, publiés ou non, émanant des établissements d'enseignement et de recherche français ou étrangers, des laboratoires publics ou privés. 
High Frequency Oscillations and spikes running down after SEEG-guided thermocoagulations in the epileptogenic network of periventricular nodular heterotopia

Julia Scholly *1,4, Francesca Pizzo *2,4, Alexander Timofeev ${ }^{3}$, Maria Paola Valenti-Hirsch ${ }^{1}$, Irène Ollivier ${ }^{3}$, François Proust ${ }^{3}$, Nicolas Roehri ${ }^{2}$, Christian-George Bénar ${ }^{2}$, Edouard Hirsch ${ }^{1}$, Fabrice Bartolomei ${ }^{2,4}$

1 University of Strasbourg, Epilepsy Unit, Hautepierre Hospital, Strasbourg, France,

2 Aix Marseille University, Institut de Neurosciences des Systèmes, Inserm U1106, Marseille, France, 3 University of Strasbourg, Department of Neurosurgery, Hautepierre Hospital, Strasbourg, France, 4 Aix Marseille University, Service de Neurophysiologie Clinique, Hôpital Timone, Marseille, France.

*These authors contributed equally to this work

Corresponding author:

Julia Scholly, MD, PhD

Service de Neurologie, Hôpital Hautepierre,

1 avenue Molière, 67098 Strasbourg, France.

Tel.: +33 388128562; fax: +33 388128533;

E-mail address: julia.scholly@chru-strasbourg.fr 


\begin{abstract}
Objective: Epilepsy associated with Periventricular Nodular Heterotopia (PNH) is characterized by complex relationships between the heterotopic and the normotopic cortex during the interictal state and at seizure onset. High frequency oscillations (HFO) have been proposed as a marker of epileptogenicity that might reflect disease activity. The effects of thermocoagulations on epileptogenicity in this context remain unknown. We aimed to investigate the interictal HFO- and spike profiles of different cortical structures before and after two consecutive SEEG-guided thermocoagulations, in correlation with seizure outcome, in a patient with $\mathrm{PNH}$-related drugresistant epilepsy.
\end{abstract}

Methods: The epileptogenic zone (EZ) was defined by SEEG analysis based on the Epileptogenicity Index. Interictal spikes, ripples $(80-250 \mathrm{~Hz})$ and fast ripples $(F R, 250-330 \mathrm{~Hz})$ were analyzed within the heterotopia, the temporal neocortex and the hippocampus.

Results: The SEEG recordings revealed a distributed EZ involving the heterotopia and the posterior temporal neocortex. Both structures were targeted by thermocoagulations. Background spikes, ripples and FR-rates were significantly higher in PNH compared to the normotopic cortex. A drastic reduction of spikes (by over $80 \%$ ) and absence of $\mathrm{FR}$ were demonstrated both in the $\mathrm{PNH}$ and in the neocortex during the second SEEG exploration 6 months after the first thermocoagulation, whereas no significant difference was observed in the posterior hippocampus. Ripples were significantly reduced by the first and suppressed by the second thermocoagulation within the three structures. Seizures relapsed after two months but decreased in frequency after the first thermocoagulation. Sustained seizure-freedom was achieved only after the second procedure.

Conclusions: Our data demonstrate the running down of interictal HFO and spikes within the epileptogenic network following thermocoagulations of heterotopic and normotopic sites involved at seizure onset. This dynamics was in good correlation with significantly improved seizure control.

Significance: Combination of ictal and different interictal markers of epileptogenicity, including HFO and spike analysis, is important to get the full picture of the epileptogenic zone and could help to evaluate the disease activity.

Key words: High Frequency Oscillations; Periventricular Nodular Heterotopia; SEEG; Epileptogenicity; Thermocoagulations 


\section{Introduction}

Drug-resistant epilepsy associated with Periventricular Nodular Heterotopia (PNH) is characterized by complex electrophysiologic relationships between the heterotopic and normotopic cortex during the interictal state and at seizure onset. Distinct epileptogenic networks might be identified, where either the PNH or the cortical areas, or both can be the leader structures (Pizzo et al., 2017)(Mirandola et al., 2017)

Stereoelectroencephalography (SEEG)-guided thermocoagulation is indicated when conventional surgical resection of the seizure-generating area is not possible. It can represent a specific treatment option targeting PNH when SEEG recordings show the PNH to be crucially involved in the Epileptogenic Zone (EZ) (Cossu et al., 2014).

High frequency oscillations (HFO) have been proposed as a marker of epileptogenicity that might reflect disease activity (Zijlmans et al., 2009) and the removal of brain tissue showing HFOs correlated with good surgical outcome (Jacobs et al., 2010).

To the best of our knowledge, the effects of thermocoagulation on the electrophysiological markers of epileptogenicity have not been explored so far. We aimed to investigate the interictal HFO- and spike profiles of different cortical structures before and after two consecutive SEEG-guided thermocoagulations, in correlation with seizure outcome, in a patient with PNH-related drugresistant epilepsy.

\section{Methods}

\subsection{Subject and clinical setting}

We prospectively studied a case of a 21-year-old right-handed female with unilateral PNH-related drug-resistant epilepsy who underwent the first SEEG recording to investigate the hypothesis of left temporo-parietal epileptogenic zone and the possible involvement of the heterotopia during seizures. MRI displayed a single heterotopic nodule in the left atrium.

Intracerebral multiple contact electrodes (Alcis, France) were implanted bilaterally (14 in the left and 2 in the right hemisphere), using a frameless stereotactic surgical robot ROSA ${ }^{\mathrm{TM}}$. The placement of electrodes was guided by the hypotheses about EZ localization, based on available non-invasive data. Cranial CT scan was then performed to verify the absence of any complication and the spatial accuracy of the implantation. CT/MRI data co-registration using free software (FreeSurfer, dcm2nii, MRICron, FSL and 3D Slicer) was performed to obtain 3D-reconstructions of patient's brain with electrodes, with automatic recognition applied for the accurate anatomical localization of each electrode contact (SEEG Assistant, Fig.1 A\&B).

The EZ was defined by visual and quantitative SEEG-signal analysis using the Epileptogenicity Index (EI)(Bartolomei et al., 2008).

Radiofrequency-thermocoagulations (TC) were performed at the end of the SEEG recording. The contacts used for TC were selected according to the following criteria: (1) contacts sampling structures that belong to the EZ, as defined by El analysis and /or (2) location within or at the MRIvisible borders of the PNH. The thermolesions of around $100 \mathrm{~mm} 3$ per target were produced between 2 contiguous contacts of the selected electrodes ( 3 to $5 \mathrm{~W}$ within 30 seconds).

The second SEEG was carried out 6 months later with objective to perform thermocoagulation of the residual heterotopia as well as additional thermolesions of the neocortical parts of the EZ network. Three depth electrodes were implanted in the left hemisphere. The TC procedure was preceded and followed by $24 \mathrm{~h}$ of video-SEEG recordings respectively. Control brain MRIs were performed at $24 \mathrm{~h}$ 
hours and 3 months after the thermocoagulations. Seizure outcome was assessed according to the Engel classification.

\subsection{SEEG-signal analysis}

Signals were recorded on a Natus system (sampling at $1024 \mathrm{~Hz}$ with 16 bits resolution; a hardware high-pass filter (cut-off $=0.16 \mathrm{~Hz}$ at $3 \mathrm{~dB}$ ), and an antialiasing low-pass filter (cut-off $=340 \mathrm{~Hz}$ ). Channels showing artifacts were excluded from analysis. All signal analyses were computed using the Anywave software (Colombet et al., 2015).

A quantitative analysis of the EZ was performed using the "Epileptogenicity Index" (Bartolomei et al., 2008). The El combines analysis of both spectral and temporal features of SEEG signals, respectively, related to the propensity of a brain area to generate fast discharges $(12.4-127 \mathrm{~Hz})$, and to the earliness of involvement of this area in the seizure. A normalized El value is used, ranging from 0 to 1 . If there is no involvement of the brain structure, the $\mathrm{El}=0$ (no epileptogenicity) whereas if the brain structure generates a rapid discharge and the time to seizure onset is minimal, the $\mathrm{EI}=1$ (maximal epileptogenicity). An El value of 0.4 was set as a threshold to define a structure as epileptogenic, based on the previous study on epileptogenicity of developmental lesions (Aubert et al., 2009). El values were computed and averaged for all invasively recorded seizures $(n=9)$.

Interictal spikes and HFO (ripples $(80-250 \mathrm{~Hz})$ and fast ripples (FR, 250-330Hz)) were analyzed during $5 \mathrm{~min}$ of NREM II-III sleep on channels within the heterotopia, the overlying temporal neocortex and the hippocampus. The non-heterotopic cortical sites will be further referred to as "normotopic cortex". The HFOs were automatically detected using the Delphos HFOs detector, based on the ZHO method, which aims at flattening the frequency spectrum to enhance the fast oscillations while preserving an optimal signal to noise ratio at each frequency (Roehri et al., 2016). They were then visually checked for morphological inspection and validation. Spike, ripple and FR rates (number events/minute) were calculated and then compared for each brain region and for each period of interest. Three periods of interest were analyzed: TO (background), during the $1^{\text {st }}$ SEEG before the TC procedure; T1, during the $2^{\text {nd }}$ SEEG 6 months later; T2, during the $2^{\text {nd }}$ SEEG $24 \mathrm{~h}$ after the TC procedure. We used Kruskal-Wallis test to check statistical significance ( $p$-value $\leq 0.05$, MATLAB).

\section{Results}

3.1 The ictal SEEG signal analysis revealed distributed, "normo-heterotopic" epileptogenic zone, involving the heterotopia and the overlying posterior temporal neocortex, both characterized by a high degree of epileptogenicity (Fig 1C, D\&E). Both structures (10 heterotopic and 6 neocortical contacts) were targeted by SEEG-thermocoagulations. The hippocampus was spared, being a part of the propagation network. Control MRI showed a small residue of heterotopia (Fig. 1F). Its complete ablation was achieved by the $2^{\text {nd }}$ TC procedure. Additional thermolesions were produced on 8 contacts in posterior temporal neocortex.

\subsection{The interictal SEEG signal analysis}

Spikes, ripples and fast ripples rates in the three regions of interest as well as their temporal dynamics are reported in Fig 2. Background spikes, ripples and FR rates were significantly higher in the heterotopic cortex compared to the adjacent mesial or lateral normotopic cortices. A drastic reduction of spikes (by over $80 \%$ ) and absence of FR were demonstrated in the $\mathrm{PNH}$ and in the posterior temporal neocortex 6 months after the 1st thermocoagulation, whereas no significant difference was observed in the posterior hippocampus that was not thermocoagulated. Ripple rates were reduced by $97 \%$ within the $\mathrm{PNH}$, by $67 \%$ within the hippocampus and by $59 \%$ in the temporal 
neocortex. Residual ripples were suppressed by the 2nd thermocoagulation within the all three structures. No difference in spike rates has been observed after the $2^{\text {nd }} \mathrm{TC}$ in the neocortical and hippocampal contacts, whereas spikes were abolished in PNH contacts.

\subsection{Clinical course}

After a seizure-free period of 2 months following the $1^{\text {st }}$ thermocoagulation, seizures relapsed but decreased in frequency by $90 \%$ (from 50 to 5 per month). Sustained seizure-freedom was achieved after the $2^{\text {nd }} \mathrm{TC}$ procedure, with seizure outcome corresponding to Engel class I at the last control (1.5 years).

\section{Discussion}

Our data demonstrate for the first time the running down of interictal HFO and spikes within the epileptogenic network following thermocoagulations of the heterotopic cortex and of the related normotopic sites involved at seizure onset. The direct lesional impact of thermocoagulations was major in the heterotopia but quite limited in the temporal neocortex. Thus, the observed global and graduate decrease of epileptogenicity likely corresponding to the running down phenomenon could reflect plastic changes due to the reversibility of secondary epileptogenesis process at this stage (Morrell and de Toledo-Morrell, 1999).

This dynamics of spike and ripple rates was in good correlation with significantly improved seizure control suggesting that in this case, the disease activity was well mirrored by different interictal epileptogenicity markers.

We found globally a good correlation between the interictal and ictal epileptogenicity markers. The structures belonging to the EZ - heterotopia and the posterior temporal neocortex - exhibited high ripple and spike rates that were drastically reduced by thermocoagulations, whereas there was no major involvement of the posterior hippocampus, interictally or during seizures, and no modification of hippocampal spikes following TC.

Looking more in detail, however, heterotopia exhibited significantly higher spike-, ripple- and in particular FR-rates, compared to the normotopic sites, similarly to focal cortical dysplasia (FerrariMarinho et al., 2015). Notwithstanding, both the PNH and the neocortex showed maximal epileptogenicity at seizure onset. There was thus some dissociation between regions showing prominent interictal FR-activity and those showing high epileptogenicity, as already reported for interictal spikes (Bartolomei et al., 2016), suggesting that these markers alone might have limited sensitivity in delineating the EZ.

The pathophysiological role of HFO generated by heterotopia and other neurodevelopmental malformations needs to be clarified. Not always concordant findings are available in the literature (Ferrari-Marinho et al., 2015)(Jacobs et al., 2009)(Roehri et al., 2018)(Pizzo et al., 2017) and no large series were described until now. The high rates of ripples, FR and spikes in heterotopia could be due to other factors, e.g. to the particular histological or functional organization of nodules (Hannan et al., 1999), not strictly reflecting epileptogenicity. Potential impact of physiological HFO should also be considered. There is actually no consensus on how to distinguish physiological from pathological HFO. Region-specific normative values for physiological HFO rates have been recently proposed (Frauscher et al., 2018). Whether these thresholds are applicable to cortical developmental malformations such as heterotopia remains to be investigated. Notwithstanding, one might expect that HFO representing biomarkers of epileptogenicity would run down following ablation or inactivation of the epileptogenic zone, as observed in our case. 
Regarding the role of the $\mathrm{PNH}$ in the epileptogenesis, some parallel could be made with the hypothalamic hamartoma-associated epilepsy, characterized by dynamic ictal network organization, with possible causal directionality in relationships between the hamartoma and the cortex or widespread epileptogenesis from the start (Scholly et al., 2017).

In this respect, our study underlines the importance of combination of ictal (Epileptogenicity Index) and different interictal biomarkers of epileptogenicity, including HFO and spike analysis, to get the full picture of the epileptogenic zone.

\section{Declarations of interest: none}

\section{References}

Aubert, S., Wendling, F., Regis, J., McGonigal, A., Figarella-Branger, D., Peragut, J.C., Girard, N., Chauvel, P., Bartolomei, F., 2009. Local and remote epileptogenicity in focal cortical dysplasias and neurodevelopmental tumours. Brain 132, 3072-3086. https://doi.org/10.1093/brain/awp242

Bartolomei, F., Chauvel, P., Wendling, F., 2008. Epileptogenicity of brain structures in human temporal lobe epilepsy: A quantified study from intracerebral EEG. Brain 131, 1818-1830. https://doi.org/10.1093/brain/awn111

Bartolomei, F., Trébuchon, A., Bonini, F., Lambert, I., Gavaret, M., Woodman, M., Giusiano, B., Wendling, F., Bénar, C., 2016. What is the concordance between the seizure onset zone and the irritative zone? A SEEG quantified study. Clin Neurophysiol 127, 1157-1162. https://doi.org/10.1016/j.clinph.2015.10.029

Colombet, B., Woodman, M., Badier, J.M., Bénar, C.G., 2015. AnyWave: A cross-platform and modular software for visualizing and processing electrophysiological signals. J. Neurosci. Methods 242, 118-126. https://doi.org/10.1016/j.jneumeth.2015.01.017

Cossu, M., Fuschillo, D., Cardinale, F., Castana, L., Francione, S., Nobili, L., Lo Russo, G., 2014. StereoEEG-guided radio-frequency thermocoagulations of epileptogenic grey-matter nodular heterotopy. J. Neurol. Neurosurg. Psychiatry 85, 611-617. https://doi.org/10.1136/jnnp-2013305514

Ferrari-Marinho, T., Perucca, P., Mok, K., Olivier, A., Hall, J., Dubeau, F., Gotman, J., 2015. Pathologic substrates of focal epilepsy influence the generation of high-frequency oscillations. Epilepsia 56, 592-598. https://doi.org/10.1111/epi.12940

Frauscher, B., von Ellenrieder, N., Zelmann, R., Rogers, C., Nguyen, D.K., Kahane, P., Dubeau, F., Gotman, J., 2018. High-Frequency Oscillations in the Normal Human Brain. Ann. Neurol. 84, 374-385. https://doi.org/10.1002/ana.25304

Hannan, A.J., Servotte, S., Katsnelson, A., Sisodiya, S., Blakemore, C., Squier, M., Molnár, Z., 1999. Characterization of nodular neuronal heterotopia in children. Brain 122, 219-238. https://doi.org/10.1093/brain/122.2.219

Jacobs, J., Levan, P., Chtillon, C.D., Olivier, A., Dubeau, F., Gotman, J., 2009. High frequency oscillations in intracranial EEGs mark epileptogenicity rather than lesion type. Brain 132, 10221037. https://doi.org/10.1093/brain/awn351

Jacobs, J., Zijlmans, M., Zelmann, R., Chatillon, C.É., Hall, J., Olivier, A., Dubeau, F., Gotman, J., 2010. High-frequency electroencephalographic oscillations correlate with outcome of epilepsy surgery. Ann. Neurol. 67, 209-220. https://doi.org/10.1002/ana.21847 
Mirandola, L., Mai, R.F., Francione, S., Pelliccia, V., Gozzo, F., Sartori, I., Nobili, L., Cardinale, F., Cossu, M., Meletti, S., Tassi, L., 2017. Stereo-EEG: Diagnostic and therapeutic tool for periventricular nodular heterotopia epilepsies. Epilepsia 58, 1962-1971. https://doi.org/10.1111/epi.13895

Morrell, F., deToledo-Morrell, L., 1999. From mirror focus to secondary epileptogenesis in man: an historical review. Adv Neurol 81, 11-23.

Pizzo, F., Roehri, N., Catenoix, H., Medina, S., McGonigal, A., Giusiano, B., Carron, R., Scavarda, D., Ostrowsky, K., Lepine, A., Boulogne, S., Scholly, J., Hirsch, E., Rheims, S., Bénar, C.-G.G., Bartolomei, F., 2017. Epileptogenic networks in nodular heterotopia: A stereoelectroencephalography study. Epilepsia 58, 1-12. https://doi.org/10.1111/epi.13919

Roehri, N., Lina, J.M., Mosher, J.C., Bartolomei, F., Benar, C.G., 2016. Time-Frequency Strategies for Increasing High-Frequency Oscillation Detectability in Intracerebral EEG. IEEE Trans. Biomed. Eng. 63, 2595-2606. https://doi.org/10.1109/TBME.2016.2556425

Roehri, N., Pizzo, F., Lagarde, S., Lambert, I., Nica, A., McGonigal, A., Giusiano, B., Bartolomei, F., Bénar, C.-G., 2018. High-frequency oscillations are not better biomarkers of epileptogenic tissues than spikes. Ann. Neurol. 83, 84-97. https://doi.org/10.1002/ana.25124

Scholly, J., Staack, A.M., Kahane, P., Scavarda, D., Régis, J., Hirsch, E., Bartolomei, F., 2017. Hypothalamic hamartoma: Epileptogenesis beyond the lesion? Epilepsia 58 Suppl 2, 32-40. https://doi.org/10.1111/epi.13755

Zijlmans, M., Jacobs, J., Zelmann, R., Dubeau, F., Gotman, J., 2009. High-frequency oscillations mirror disease activity in patients with epilepsy. Neurology 72, 979-986.

https://doi.org/10.1212/01.wnl.0000344402.20334.81 
A

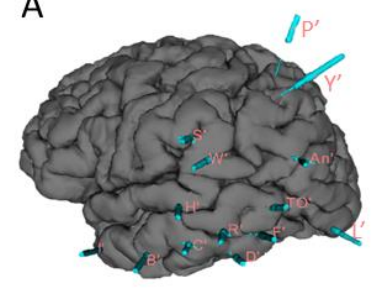

C

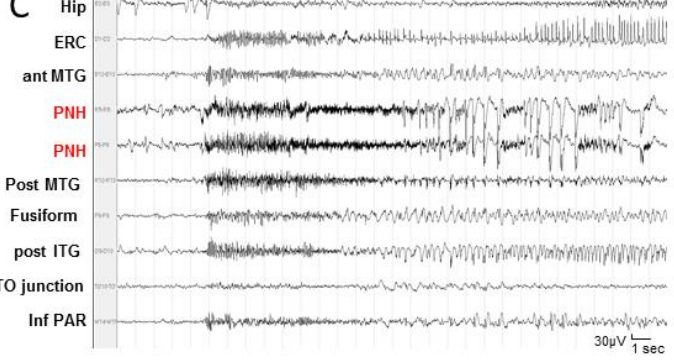

ouv $\frac{1 \text { sec }}{5}$
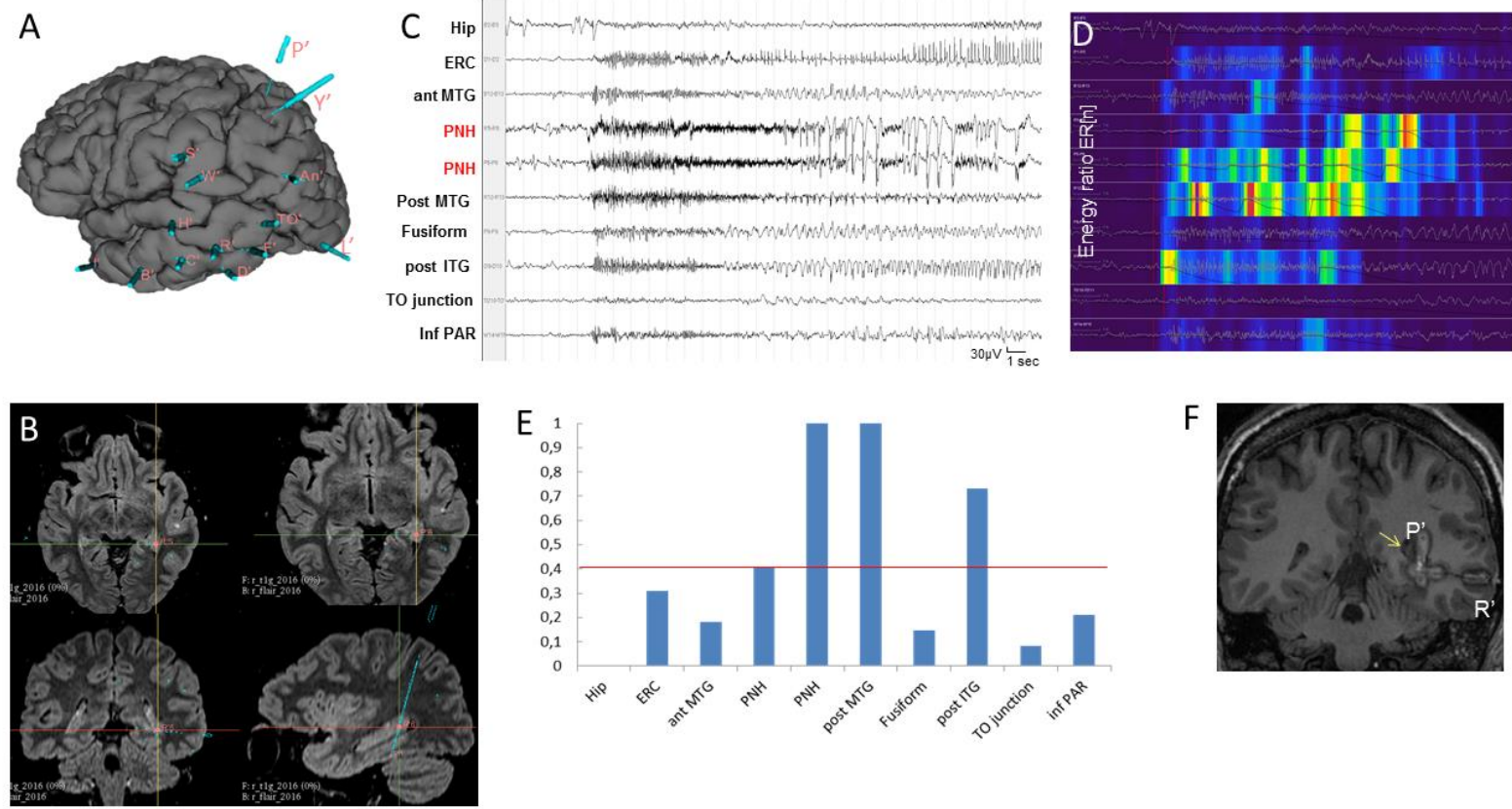

F

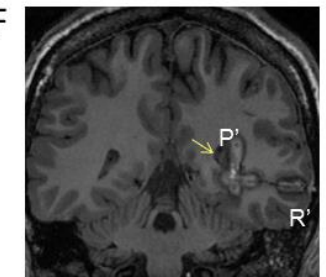

\section{Figure 1. Ictal SEEG findings and organization of the epileptogenic zone.}

A. Patient's left hemisphere surface volume rendering with implanted SEEG electrodes. Reconstructed electrode images (blue) correspond to real trajectories and entry points. Electrode I' sampled the mesial and lateral aspects of the temporal pole; electrodes $\mathrm{B}^{\prime}$ and $\mathrm{C}^{\prime}$ explored the anterior and posterior hippocampus (internal contacts) and the middle and posterior aspects of the middle temporal gyrus (MTG); electrode $\mathrm{H}^{\prime}$ recorded the posterior inferior insula, the Heschl gyrus (BA41/42) and the posterior superior temporal gyrus (BA22); electrode $\mathrm{R}^{\prime}$ recorded the retrosplenial gyrus (contacts 1-3), anterior portion of the periventricular nodular heterotopia (PNH, contacts 5,6) and the posterior MTG (contacts 11-14); electrode $\mathrm{P}^{\prime}$ explored the posterior fusiform gyrus (contacts 1,2), the posterior portion of the PNH (contacts 5-8) and the superior parietal lobule (contacts 16-18); electrode D' sampled the parahippocampal, the fusiform and the inferior temporal gyri (ITG); electrode $\mathrm{F}^{\prime}$ explored the posterior aspects of the fusiform and the ITG respectively; electrode TO' sampled the mesial temporo-occipital junction and the posterior MTG, adjacent to the angular gyrus; electrode L' explored the lingual and the inferior occipital gyri; electrode An' sampled the inferior part of the occipito-parietal sulcus and the angular gyrus (BA19); electrode $Y^{\prime}$ recorded the posterior insula (internal contacts) and the superior aspect of the angular gyrus (lateral contacts); electrode $\mathrm{W}^{\prime}$ explored the precuneus and the supramarginal gyrus (BA 40); electrode $\mathrm{S}^{\prime}$ sampled the posterior cingulum and the anterior superior aspect of the supramarginal gyrus (BA 39).

B. Position of electrode contacts sampling the PNH ( $\left.\mathrm{R}^{\prime} 5-6, \mathrm{P}^{\prime} 5-8\right)$ shown on 3D-reconstructions of brain MRI with electrodes. C. SEEG recordings of a habitual seizure that starts simultaneously from the heterotopia (PNH) and different cortical subregions of the left temporal lobe, with tonic discharge affecting the PNH and the posterior middle and inferior temporal gyri. D. Changes in energy ratio of high frequencies (ER[n]) in the above mentioned SEEG signals are disclosed with a color scale. Early increase in ER is seen both in the heterotopic and normotopic (posterior temporal) structures. E. The Epileptogenicity Index values (EI) obtained in each analyzed structure in this seizure, demonstrating maximal epileptogenicity in the PNH and in the posterior temporal neocortex. The red line marks the 0.4 value considered as a threshold to define a region as epileptogenic. $F$. Brain MRI 3mo after the 1st TC showing the thermolesions within the PNH and the overlying neocortex. Note a small residue of heterotopic cortex mesially from the lesional contacts of the electrode $\mathrm{P}^{\prime}$ (yellow arrow). 

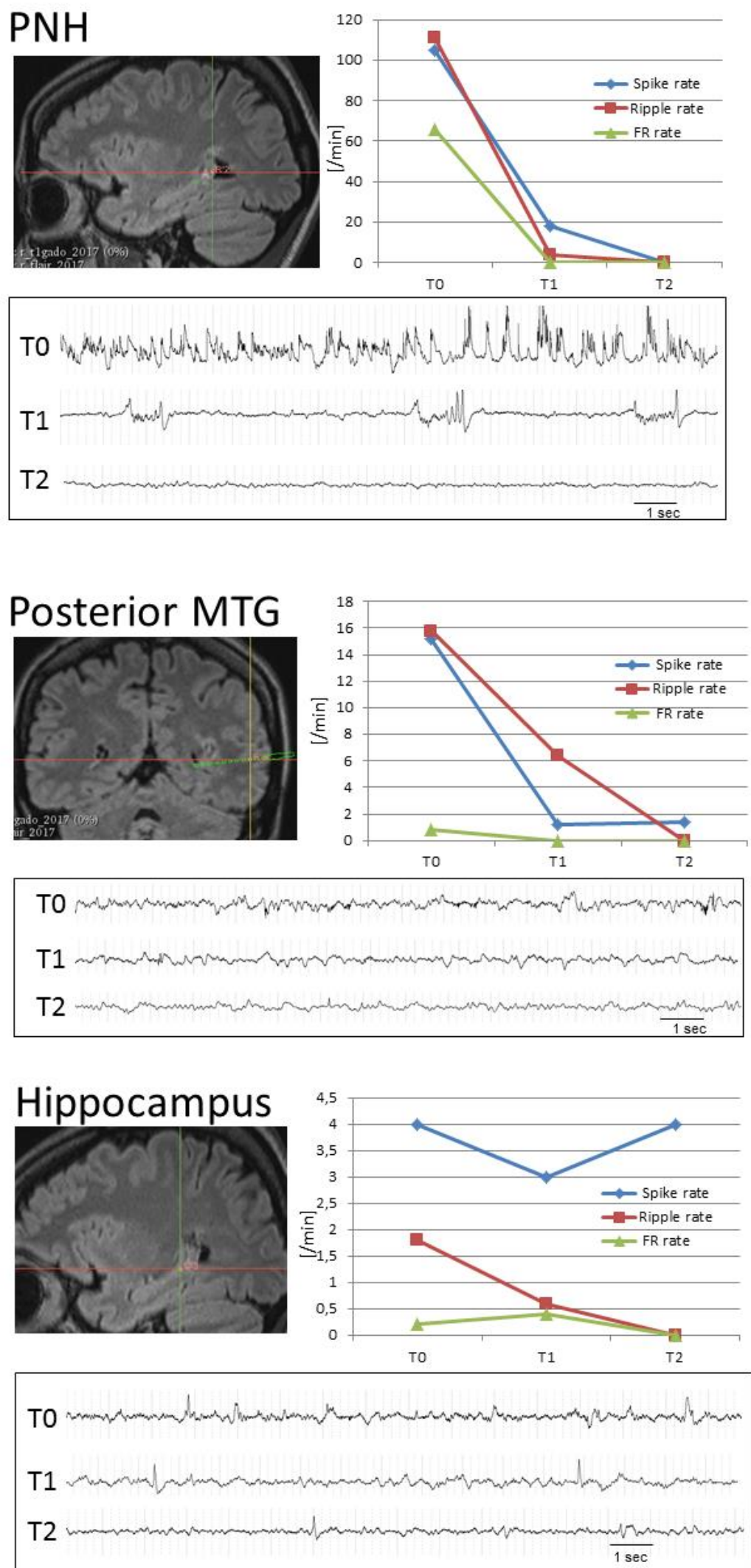

Figure 2. Interictal markers of epileptogenicity in heterotopic and normotopic cortical structures. Temporal dynamics of the spike-, ripple- and fast ripple (FR) -rates (top right diagrams) and the interictal SEEG signal aspect (bottom boxes) are shown within the heterotopic cortex, the hippocampus and the posterior temporal neocortex respectively, before (TO) and after $(\mathrm{T} 1, \mathrm{~T} 2)$ two consecutive SEEG-thermocoagulation procedures. Top left: Representative electrode contact within the structure of interest displayed on 3Dreconstructions of patient's brain MRI. 